\title{
A Fully Preemptive Multiprocessor Semaphore Protocol for Latency-Sensitive Real-Time Applications
}

\author{
Björn B. Brandenburg \\ Max Planck Institute for Software Systems (MPI-SWS)
}

\begin{abstract}
Independence preservation, a property in real-time locking protocols that isolates latency-sensitive tasks from delays due to unrelated critical sections, is identified, formalized, and studied in detail. The key to independence preservation is to ensure that tasks remain fully preemptive at all times. For example, on uniprocessors, the classic priority inheritance protocol is independence-preserving. It is shown that, on multiprocessors, independence preservation is impossible if job migrations are disallowed. The $O(m)$ independencepreserving protocol (OMIP), a new, asymptotically optimal binary sempahore protocol based on migratory priority inheritance, is proposed and analyzed. The OMIP is the first independence-preserving, real-time, suspension-based locking protocol for clustered job-level fixed-priority scheduling. It is shown to benefit latency-sensitive workloads, both analytically by means of schedulability experiments, and empirically using response-time measurements in LITMUS ${ }^{R T}$.
\end{abstract}

\section{Introduction}

A frequent, but challenging requirement in the design and implementation of practical real-time systems is the need for predictable, mutually exclusive access to shared resources such as I/O ports, network links, message buffers, or other shared data structures. To this end, virtually all modern realtime operating systems (RTOSs) offer some sort of locking primitives, most commonly binary semaphores (or mutexes), under which tasks suspend when trying to access already locked resources. The defining characteristics of a real-time semaphore protocol are that it (i) must guarantee bounded delays for all real-time tasks - which at times requires lockholding tasks to be scheduled despite the presence of (otherwise) higher-priority tasks [31, 34]—while (ii) simultaneously ensuring that urgent, high-priority tasks are not unduly delayed by unrelated critical sections in lower-priority tasks.

In the uniprocessor case, the real-time locking problem has long been solved: the three classic uniprocessor realtime semaphore protocols, namely the priority-inheritance protocol (PIP) [31, 34], the priority-ceiling protocol (PCP) [31, 34], and the stack resource policy (SRP) [3], fully satisfy requirements (i) and (ii). Unfortunately, the same cannot be said of the multiprocessor case. While numerous protocols satisfying requirement (i) have been developed for various scheduling approaches (reviewed below), we show in this paper that all existing protocols fail to meet the second requirement when confronted with latency-sensitive workloads (i.e., if some tasks have near-zero tolerance for scheduling delays) under partitioned scheduling (where each task is statically assigned to a processor and each processor is scheduled individually). In other words, there exist demanding, but common hard real-time workloads that currently simply cannot be provisioned on multiprocessors under partitioned scheduling (which is widespread in practice) due to the lack of appropriate synchronization primitives.

The primary contribution of this paper is a solution to this problem: we present the design, analysis, implementation, and evaluation of the first multiprocessor real-time semaphore protocol applicable to partitioned scheduling (and other policies) that guarantees that latency-sensitive applications are never delayed by unrelated lower-priority tasks.

The problem. To understand why existing multiprocessor semaphore protocols fail, first consider why the classic uniprocessor protocols $d o$ work. For example, consider the de facto industry standard of preemptive fixed-priority (FP) scheduling in conjunction with the PIP, and let $T_{h}$ denote the highest-priority task in the system. Priority inheritance $[31,34]$ famously addresses requirement (i) by temporarily raising the priority of a lock-holding task to that of the highest-priority waiter (if any). One major advantage of this approach is that task $T_{h}$ is never delayed by critical sections associated with resources shared only among lower-priority tasks, since such critical sections always execute at a lower priority. As a result, such unrelated critical sections may be of arbitrary length without affecting the temporal correctness of $T_{h}$. This isolation property, which we formalize as "independence preservation" in Sec. 3, crucially depends on the fact that lock-holding tasks remain fully preemptive.

Now consider the most straightforward (and widespread) multiprocessor extension of FP scheduling, namely partitioned fixed-priority (P-FP) scheduling, together with the multiprocessor priority-ceiling protocol (MPCP) [30, 31], a classic shared-memory semaphore protocol for P-FP scheduling. Under partitioned scheduling, it is particularly difficult to ensure that lock-holders are scheduled when remote tasks are blocked; ${ }^{1}$ the MPCP_-and all real-time semaphore protocols for partitioned scheduling developed since-employ priority boosting, that is, they simply raise the priority of lock-holding tasks above any "normal" priority, thereby ensuring that lock-holding tasks are never

\footnotetext{
${ }^{1}$ Priority inheritance is ineffective under partitioning, e.g., see [8, 13]
} 
preempted by non-lock-holding tasks. While effective, this simple technique has the unfortunate consequence that lockholding tasks are effectively no longer fully preemptive: even the highest-priority task $T_{h}$ cannot preempt all other tasks at all times. As a result, there is no isolation: the temporal correctness of any task depends on the maximum critical section length of any other task (assigned to the same processor). Crucially, this is unacceptable for latency-sensitive workloads, where lower-priority tasks must not affect the correctness of the highest-priority tasks.

The described phenomenon is not specific to the MPCP, but rather a consequence of priority boosting. While priority boosting can be acceptable if all critical sections can be ascertained with high confidence to always be "short enough," latency-sensitive workloads with highly heterogeneous timing requirements fundamentally require high-priority tasks to be shielded from unrelated critical sections. Such workloads are common in practice. For instance, in the automotive domain, it is not unusual for real-time constraints to span three orders of magnitude, ranging from a few milliseconds for high-frequency control tasks up to one second for less urgent tasks (e.g., see [37]). However, in a task with a period of 1000 milliseconds, it is not unreasonable to encounter critical sections with worst-case lengths in excess of one millisecond, especially in embedded systems with relatively slow processors. Clearly such a critical section cannot be permitted to delay latency-sensitive tasks (e.g., those with a one-millisecond period). However, as we discuss next, none of the real-time semaphore protocols for partitioned scheduling proposed to date can actually guarantee this-a major oversight that we rectify in this paper.

Related work. Multiprocessor semaphore protocols are typically designed for, and tightly integrated with, a specific scheduling policy. Most relevant to our work are prior semaphore protocols for partitioned scheduling. The first such protocols were proposed by Rajkumar et al. [30-32], who designed the aforementioned MPCP and the distributed $P C P$ for P-FP-scheduled systems. In later work, Block et al. [7] proposed the flexible multiprocessor real-time locking protocol (FMLP), and an improved version for partitioned scheduling, termed FMLP $^{+}$, has since been developed in [8]. In recent work, Nemati et al. [28] proposed a semaphore protocol that simplifies the consolidation of independently developed (legacy) applications onto P-FP. scheduled multicore platforms. Crucially, all of the just-cited protocols employ priority boosting and are thus not suitable for latency-sensitive workloads, as explained above.

In work focused on global scheduling, under which (conceptually) all processors serve a single ready queue and tasks may migrate freely among cores, several semaphore protocols have been proposed in recent years, including a global variant of the FMLP and two extensions of the PCP [21, 27]. These protocols rely on priority inheritance [34] instead of priority boosting and allow tasks to remain fully preemptive; however, they apply only to global scheduling and cannot be easily transferred to the partitioned case, which is (more) commonly used in industrial applications (e.g., partitioning is mandated by the Autosar 4.0 standard).

Most closely related to this paper are optimality results $[8,12,13]$ pertaining to the intuitive goal of "minimal blocking" in multiprocessor real-time semaphore protocols under job-level fixed-priority (JLFP) scheduling, a broad category of schedulers that includes the FP and earliest-deadline first (EDF) policies. Specifically, recent work [12] investigated asymptotic bounds on maximum priority-inversion blocking (pi-blocking), which (intuitively) occurs whenever a high-priority task is delayed by a lowerpriority one. Surprisingly, there exist two classes of schedulability analysis, called suspension-aware and suspensionoblivious analysis [12], resp., that give rise to different lower bounds on worst-case pi-blocking, namely $\Omega(n)$ and $\Omega(m)$, resp., where $n$ denotes the number of tasks and $m$ the number of processors. In brief, the difference arises due to how semaphore-induced self-suspensions are accounted for.

This paper pertains to the suspension-oblivious case, for which there already exists the asymptotically optimal family of $O(m)$ locking protocols (OMLP) [8, 12, 13]. In particular, the OMLP family contains semaphore-based mutual exclusion protocols for global scheduling, partitioned scheduling, and for clustered scheduling [4, 17], which is a hybrid (or generalization) of global and partitioned scheduling where tasks are statically assigned to non-overlapping subsets (or clusters) of processors, and each cluster is scheduled using a global policy. We revisit these protocols, denoted as $P$ OMLP, G-OMLP, and C-OMLP, resp., in greater detail in Sec. 2. However, note that the OMLP family decidedly does not solve the problem addressed in this paper, which is to support latency-sensitive workloads under clustered scheduling, since the priority-inheritance-based G-OMLP applies only to global scheduling, whereas the P-OMLP and the C-OMLP use (a variant of) priority boosting, and thus are susceptible to potentially problematic latency increases.

A well-studied, sometimes-preferable alternative to semaphores are non-preemptive spinlocks, in which blocked jobs busy-wait by executing a delay loop (e.g., see [7, 8, $18,25])$. While busy-waiting conceptually wastes processor cycles, non-preemptive spinlocks are an attractive choice in practice due to their simplicity and low overheads $[8,15]$. Unfortunately, non-preemptive spinlocks can cause unacceptable delays for latency-sensitive workloads because tasks may remain non-preemptive for prolonged times. While it is possible to reduce the negative effects by allowing spinning tasks to remain preemptive (e.g., see [2, 20, 35]), critical sections are still executed non-preemptively in such spinlocks and thus have a major impact on latency, not unlike the priority-boosting of critical sections under the MPCP.

The need for non-preemptive execution can be avoided altogether with helping mechanisms (e.g., see [36]), where instead of executing a delay loop, "spinning" tasks attempt 
to complete the critical sections of preempted lock holders. However, such helping-based approaches have two limitations in practice. First, they are considerably more complicated than regular spinlocks, which negates one of the key reasons to use spinlocks in the first place, and second, they are less general than regular locks. That is, "helping" cannot be offered as a generic OS primitive, but must be integrated individually into each application because critical sections must be implemented in a "helping-aware" manner such that they can be posted to a queue of pending operations.

Finally, in work aimed at temporal isolation among tasks in mixed real-time/non-real-time environments, Faggioli et al. [24] presented the multiprocessor bandwidthinheritance protocol (MBWI), a scheduler-agnostic locking protocol based on preemptive spinning. Notably, to ensure progress, the MBWI allows lock-holding tasks to migrate to processors on which tasks are waiting for the lock, an effective technique that we reuse in Sec. 3.

Contributions. We provide a precise definition of independence preservation, which captures the idea that "tasks should be isolated from unrelated critical sections," and provide evidence of its considerable practical importance (Sec. 3). We show in Sec. 4.1 that this desirable property is impossible on multiprocessors unless jobs may (temporarily) migrate among all processors. Based on this observation, we present in Sec. 4.2 our main contribution, the $O(\mathrm{~m})$ independence-preserving locking protocol (OMIP) for clustered scheduling, and show that it is optimal within a factor of approximately two under suspension-oblivious analysis (Sec. 4.3). Finally, in Sec. 5, we report on response-time measurements and schedulability experiments that show the OMIP to greatly benefit latency-sensitive workloads. We begin with a review of needed background.

\section{Background and Definitions}

This paper assumes the classic sporadic task model and follows the conventions from [12].

Tasks and jobs. The workload is specified as a set of $n$ sporadic tasks $\tau=\left\{T_{1}, \ldots, T_{n}\right\}$ that is to be scheduled on $m$ identical processors. We let $T_{i}$ denote a task with a worstcase per-job execution time $e_{i}$ and a minimum job separation $p_{i}$, and let $J_{i}$ denote a job of $T_{i}$. Our results do not depend on the type of deadline constraint; we assume implicit deadlines for simplicity. A task's utilization is the ratio $u_{i}=e_{i} / p_{i}$, and $\tau$ 's total utilization is given by $U=\sum_{T_{i} \in \tau} u_{i}$.

A task's slack $p_{i}-e_{i}$ describes how much delay one of its jobs can tolerate without missing its deadline. A task is latency-sensitive if its slack does not exceed the maximum critical section length of other tasks (see below).

Jobs are pending from the time when they are released until they complete. A pending job can be in one of two states: a ready job is available for execution, whereas a suspended job cannot be scheduled. A job resumes when its state changes from suspended to ready.
Scheduling. Under clustered scheduling [4, 17], processors are grouped into $\frac{m}{c}$ non-overlapping sets (or clusters) of $c$ processors each, where $C_{k}$ denotes the $k^{\text {th }}$ cluster. We require uniform cluster sizes and for simplicity assume that $m$ is an integer multiple of $c .^{2}$ Each task is statically assigned to a cluster; we let $C\left(T_{i}\right)$ denote the cluster to which task $T_{i}$ has been assigned. Partitioned $(c=1)$ and global $(c=m)$ scheduling are special cases of clustered scheduling.

Priority. Each cluster is scheduled independently using a work-conserving job-level fixed-priority (JLFP) scheduler. Under a JLFP scheduler, each job is assigned a unique base priority that does not change while it is pending. However, a locking protocol (see below) may temporarily raise the effective priority of a job to ensure its timely completion. At any point in time and w.r.t. each cluster, the $c$ highesteffective-priority ready jobs are scheduled.

JLFP scheduling is a broad category of scheduling policies that includes EDF and FP scheduling. Note that in the following, we use the terms "higher-priority" and "lowerpriority" to refer to the generic JLFP notion of a job's base priority (and not to a task's priority under FP scheduling). For illustrative purposes, we consider clustered earliestdeadline-first (C-EDF) scheduling as a representative JLFP policy. (We chose C-EDF since it is the policy actually implemented in LITMUS ${ }^{\text {RT }}$, the RTOS underlying the evaluation in Sec. 5.) We let P-EDF denote the special case of partitioned scheduling (i.e., C-EDF scheduling with $c=1$ ).

Real-time locking. Besides the $m$ processors, tasks share $r$ serially-reusable shared resources $\ell_{1}, \ldots, \ell_{r}$. Access to shared resources is governed by a locking protocol that ensures mutual exclusion by serializing conflicting requests.

A job $J_{i}$ requests a resource $\ell_{q}$ at most $N_{i, q}$ times. If $\ell_{q}$ is already in use, $J_{i}$ incurs acquisition delay until its request for $\ell_{q}$ is satisfied. In this paper, we focus on semaphore protocols, that is, jobs wait by self-suspending. Once $J_{i}$ has finished using $\ell_{q}$, its request is complete and $J_{i}$ releases $\ell_{q}$. We let $L_{i, q}$ denote the maximum critical section length, that is, the maximum time that $J_{i}$ uses $\ell_{q}$ as part of a single request (or critical section). We assume that $J_{i}$ must be scheduled in order to use $\ell_{q}$; however, the analysis in Sec. 4 remains valid even if critical sections contain suspensions (e.g., when performing I/O). For notational convenience, we define $L_{q}^{\max } \triangleq \max _{i}\left\{L_{i, q}\right\}$ and $L^{\max } \triangleq \max _{q}\left\{L_{q}^{\max }\right\}$, and require that $L_{i, q}=0$ if $N_{i, q}=0$.

We assume that tasks do not hold resources across job boundaries and that jobs request at most one resource at any time. Analysis of nested critical sections is beyond the scope of this paper and the subject of future work (see Sec. 6).

Priority inversion. Intuitively, a priority inversion exists if a high-priority job that should be scheduled is not scheduled, which is typically due to acquisition delay. However,

\footnotetext{
${ }^{2}$ If $c$ does not evenly divide $m$, there are $\left\lfloor\frac{m}{c}\right\rfloor$ clusters of size $c$ and one cluster of size $m \bmod c$. This does not affect the asymptotic bounds.
} 
priority inversion is not the same as acquisition delay: a suspended job does not suffer a priority inversion if it would not have been scheduled anyway (i.e., in the presence of higher-priority jobs) $[31,34]$. We let $b_{i}$ denote the maximum cumulative duration of priority inversion incurred by any $J_{i}$.

Recent work $[8,12]$ showed that what exactly constitutes a priority inversion (Def. 1 below) depends on how selfsuspensions are accounted for during schedulability analysis (i.e., when checking whether all jobs will meet their deadline). Accurate analysis of self-suspensions is NP-hard [33] and complicates schedulability tests considerably. Therefore, many published tests $(e . g .,[5,6])$ make the simplifying assumption that tasks never self-suspend. Such suspensionoblivious (s-oblivious) tests can still be used with semaphores by inflating each task's execution $\operatorname{cost} e_{i}$ by $b_{i}$ prior to applying the test [12]. In contrast, suspension-aware (s-aware) schedulability tests explicitly account for self-suspensions. The focus of this paper is s-oblivious analysis; the s-aware case is subject of ongoing work. S-oblivious analysis yields the following definition of "priority inversion."

Def. 1. A pending job $J_{i}$ suffers an s-oblivious priority inversion at time $t$ iff $J_{i}$ is (i) not scheduled and (ii) among the $c$ highest-base-priority pending jobs in cluster $C\left(T_{i}\right)$ [12].

Note that clause (ii) pertains only to jobs in $J_{i}$ 's assigned cluster $C\left(T_{i}\right)$ - the priorities of jobs in other clusters are irrelevant since each cluster is analyzed individually-and that it pertains to pending (and not just ready) jobs because self-suspensions are analyzed as execution time.

Priority inversions are considered to cause "blocking" because they lead to an undesirable increase in worst-case response time. To avoid confusing such locking-related delays with other uses of the word "blocking," we use the more specific term "pi-blocking" in this paper.

PI-Blocking optimality. The purpose of a real-time locking protocol is to minimize the occurrence and duration of priority inversions, which, however, cannot be avoided entirely. The significance of differentiating between s-oblivious and saware analysis is that they give rise to different lower bounds on maximum pi-blocking, formally $\max _{\tau}\left\{b_{i}\right\}$ [12]. In particular, the s-oblivious case is subject to an $\Omega(m)$ lower bound on maximum pi-blocking, whereas s-aware analysis yields an $\Omega(n)$ lower bound (where $n \geq m$ ). In other words, under soblivious analysis, there exist pathological task sets such that pi-blocking is linear in the number of processors under any semaphore protocol. To be asymptotically optimal, a locking protocol must ensure that maximum pi-blocking is always within a constant factor of $m$. In the suspension-oblivious case, $\max _{i}\left\{b_{i}\right\}=O(m)$ is hence asymptotically optimal. When stating asymptotic bounds, $L^{\max }$ and $\sum_{q} N_{i, q}$ are considered constant (i.e., not a function of $n$ or $m$ ) [12, 13]. The task execution costs $e_{i}$ are not constants, and pi-blocking is considered "bounded" only if no $b_{i}$ depends on any $e_{i}$.

The OMLP. The family of $O(m)$ locking protocols is a collection of in total five asymptotically optimal locking proto- cols for global, partitioned, and clustered JLFP scheduling $[8,12,13]$. Most relevant to this paper are the two mutual exclusion protocols for partitioned [12] and clustered scheduling [13], which we denote as P-OMLP and C-OMLP, resp. Both use priority boosting to ensure lock-holder progress, which means that the effective priority of lock-holding jobs exceeds any possible base priority. Lock-holding jobs thus are never preempted by non-lock-holding jobs, and in particular not by newly-released jobs (which cannot yet have acquired any locks at the time of their release).

While we consider clustered JLFP scheduling with arbitrary cluster sizes, we focus on the P-OMLP [12] as a baseline because it is somewhat simpler and because it suffices to reveal the limitations of priority boosting. The key difference between the two protocols is that the P-OMLP is asymptotically optimal only for $c=1$, whereas the COMLP uses a refined version of priority boosting called priority donation that allows it to cover the entire range of $1 \leq c \leq m$ [13] . For the purpose of this paper, the C-OMLP and the P-OMLP can be considered to be equivalent.

In brief, the P-OMLP works as follows. Requests for each $\ell_{q}$ are serialized with a FIFO queue $\mathrm{FQ}_{q}$. However, before a job can be enqueued in any $\mathrm{FQ}_{q}$, it must hold its processor's contention token $\mathrm{CT}_{k}$. As there is only one such token per processor, at most $m$ jobs are enqueued in any $\mathrm{FQ}_{q}$ at any time, which yields the desired $O(m)$ bound. Contention for each $\mathrm{CT}_{k}$ is managed using a per-processor priority queue $\mathrm{PQ}_{k}$. The P-OMLP is simple, easy to implement [8], ensures asymptotically optimal maximum pi-blocking, and has been shown to perform well compared to the FMLP $^{+}$and the MPCP for certain workloads [8, 13]. However, its chief limitation, which it shares with the FMLP ${ }^{+}$and the MPCP, is its reliance on priority boosting, as we show in detail next.

\section{Independence Preservation}

In this section, we first give a precise specification for the intuitive requirement that "high-priority jobs should be isolated from unrelated critical sections," and then provide evidence of its considerable practical importance.

To motivate the need for such isolation, consider the example P-OMLP schedule given in Fig. 1(a), which demonstrates that priority boosting is ill-suited to latency-sensitive workloads. In this particular example, task $T_{1}$ with parameters $e_{1}=6$ and $p_{1}=10$ is latency-sensitive since it has only four time units of slack, less than task $T_{2}$ 's maximum critical section length $\left(L_{2,1}=5\right)$. In the depicted schedule, job $J_{2}$ acquires resource $\ell_{1}$ and becomes priority-boosted shortly after its release at time 0 . Hence, job $J_{1}$ cannot preempt $J_{2}$ at time 1 and consequently misses its deadline at time 11 . Notably, $J_{1}$ does not access shared resources and is continuously the highest-priority job while it is pending - clearly it should not incur any delays, but under the P-OMLP (or any other semaphore protocol based on priority boosting), $J_{1}$ is not isolated from $J_{2}$ 's long critical section because $J_{2}$ 


\begin{tabular}{|llll}
\hline scheduled on processor & $\uparrow$ job release \\
\hline & & &
\end{tabular}

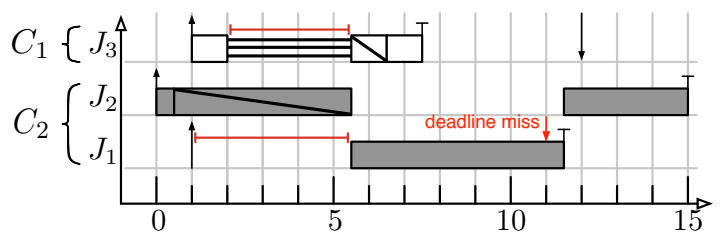

(a) Priority boosting causes pi-blocking for independent jobs.

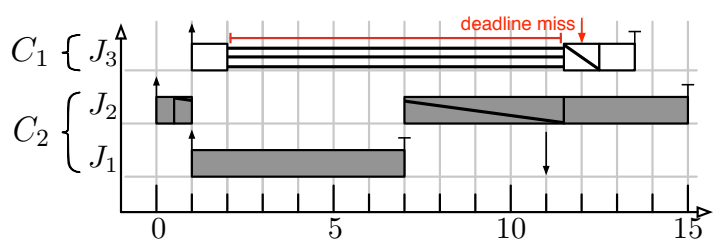

(b) Priority inheritance is ineffective across clusters.

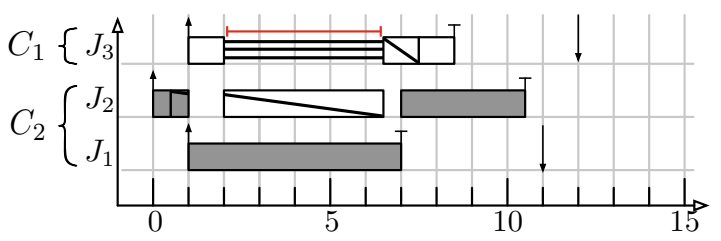

(c) Temporarily migrating $J_{2}$ to $C_{1}$ renders the task set schedulable.

Figure 1: P-EDF schedules $(c=1)$ of three tasks $T_{1}, T_{2}, T_{3}$ with execution costs $e_{1}=6, e_{2}=9, e_{3}=3$, and periods $p_{1}=10$, $p_{2}=40$, and $p_{3}=11$. Tasks $T_{2}$ and $T_{3}$ share one resource $\left(\ell_{1}\right)$, where $L_{2,1}=5$ and $L_{3,1}=1$. While P-EDF is assumed in this example, the same effect can arise under any partitioned JLFP scheduler (including P-FP). The legend applies to Fig. 2, too.

is effectively non-preemptable while accessing $\ell_{1}$.

It bears repeating that classic priority inheritance [34], which allows jobs to remain fully preemptive, is unfortunately ineffective (analytically speaking) when applied across clusters or partitions, as shown in Fig. 1(b). At time 2 , job $J_{3}$ attempts to lock $\ell_{1}$, but must wait since $J_{2}$ already holds the resource. Assuming priority inheritance, this causes $J_{2}$ 's effective priority (i.e., deadline under PEDF) to be raised from time 40 ( $J_{2}$ 's deadline) to time 12 ( $J_{3}$ 's deadline). However, $J_{1}$ has an even earlier deadline at time 11 and $J_{2}$ remains preempted. As a result, $J_{3}$ incurs unbounded pi-blocking in the sense that it is no longer possible to bound pi-blocking only in terms of critical section lengths ( $b_{3}$ depends on $e_{1}$ ). A deadline miss results.

However, a simple tweak renders this example schedulable: instead of boosting $J_{2}$ 's priority while holding $\ell_{1}$, it should simply be migrated to $J_{3}$ 's cluster $C_{1}$ when $J_{3}$ requests $\ell_{1}$. As illustrated in Fig. 1(c), this allows $J_{2}$ to complete its critical section in a timely fashion so that $J_{3}$ can meet its deadline. Notably, this is accomplished without causing any additional delay for the independent, latencysensitive job $J_{1}$, and while some pi-blocking is unavoidable when using semaphores [12], it is only incurred by the job that actually requires a shared resource ( $J_{3}$ in this example). We formalize this property as follows (first defined in [8]).

Def. 2. Let $b_{i, q}$ denote the maximum pi-blocking incurred by $J_{i}$ due to requests by any task for resource $\ell_{q}$. Under s-oblivious analysis, a locking protocol is independencepreserving if and only if $N_{i, q}=0$ implies $b_{i, q}=0$.

In other words, independence among tasks is preserved if the overall s-oblivious ${ }^{3}$ pi-blocking $b_{i}=\sum_{q} b_{i, q}$ does not depend on resources that a task does not access.

Significance. We posit that independence preservation is indispensable for many workloads common in current practice in industry, as illustrated by the following use cases.

First, if the locks acquired (only) by lower-priority tasks protect data structures of potentially unbounded size (e.g., lists or trees of unknown size), or if they serialize operations of potentially unbounded or unknown runtime (e.g., vendorprovided "black-box" functionality), then it is not possible to derive a priori bounds on the maximum duration that lower-priority tasks remain effectively non-preemptive. This renders priority-boosting-based locking protocols unviable for real-time systems that need to be certified, even if the developer is certain that all lower-priority critical sections are "short enough." Due to the lack of isolation under priority boosting, a small, but hard-to-quantify risk remains, which can pose considerable challenges in the certification process. Use of a predictable, independence-preserving locking protocol allows to categorically rule out such concerns.

Second, in open systems (i.e., in systems in which the final task set composition is unknown at design time), in RTOSs based on commodity OSs, and in real-time applications incorporating commodity libraries (e.g., for common tasks such as encryption, compression, or signal processing), locks acquired by lower-priority (or even best-effort) tasks often stem from (a large volume of) untrusted code. In this case, it is (at least economically) impossible to rule out the existence of unknown, potentially dangerous critical sections. For instance, this is a major problem in real-time Linux variants, since the Linux kernel contains millions of lines of code of varying quality (device drivers are notorious for aboveaverage defect densities [19]), and because real-time locking primitives are exported to all, potentially untrusted userspace tasks (via the pthreads library). With an independencepreserving locking protocol, tasks remain fully preemptive and it is sufficient to inspect and test only the (few) parts of the kernel actually used by latency-sensitive tasks.

Finally, current industrial practice heavily favors fully preemptive kernel designs and uses scheduling latency (i.e., how quickly a newly released high-priority job can be sched-

\footnotetext{
${ }^{3}$ Def. 2 is specific to s-oblivious analysis. For technical reasons, s-aware analysis requires a slightly weaker definition (a subject of future work).
} 
uled) as a key evaluation metric. For example, the non-profit Open Source Automation Development Lab reports longterm, large-scale scheduling latency measurements of Linuxbased real-time systems to demonstrate Linux's viability as an RTOS [29]. Similarly, commercial RTOSs are commonly advertised as sporting fully preemptive designs and negligible scheduling latencies. Case in point, a prominently advertised feature of the commercial ThreadX uniprocessor RTOS is that " $[\mathrm{a}]$ high priority thread starts responding to an external event on the order of the time it takes to perform a highly optimized ThreadX context switch" [23]. Without an independence-preserving locking protocol, it will be impossible to extend such claims to partitioned scheduling.

Taken as a whole, these examples reveal a clear need for practical real-time multiprocessor semaphore protocols that are both predictable and independence-preserving. However, in the case of partitioned and clustered scheduling, no such protocols have been proposed to date. To address this gap, we present the first design and analysis of an independence-preserving real-time semaphore protocol for clustered scheduling in the remainder of this paper.

\section{The OMIP for Clustered JLFP Scheduling}

Real-time semaphore protocols must deal with two central issues: how to avoid excessive pi-blocking when lock-holding jobs are preempted (Sec. 4.1), and how to order conflicting requests such that pi-blocking is minimized to the extent possible (Sec. 4.2). We begin with the former aspect.

\subsection{An Independence-Preserving Progress Mechanism}

As reviewed in Sec. 1, all prior real-time semaphore protocols for partitioned and clustered scheduling use variants of priority boosting to ensure lock-holder progress, which is not independence-preserving. A new progress mechanism is thus required. Interestingly, the example schedule in Fig. 1(c) contains an inter-cluster migration (i.e., job $J_{2}$ must temporarily execute in cluster $C_{1}$ despite $T_{2}$ being assigned to $C_{2}$ ), which runs counter to the idea of clustered scheduling. Such migrations are in fact unavoidable.

Theorem 1. Under clustered JLFP scheduling with $c \neq m$, it is impossible for a semaphore protocol to simultaneously (i) prevent unbounded pi-blocking, (ii) be independencepreserving, and (iii) avoid inter-cluster migrations.

Proof. Consider Fig. 1. When job $J_{3}$ issues its request at time 2, any locking protocol that does not employ intercluster migrations has only two options: either it forces $J_{2}$ to complete its critical section at the expense of delaying $J_{1}$, as shown in Fig. 1(a), or the locking protocol can isolate $J_{1}$ from any locking-related delays, as shown in Fig. 1(b). In the former case, the locking protocol is not independencepreserving, in the latter case, $J_{3}$ is subject to unbounded pi-blocking because $b_{3}$ then depends on $J_{1}$ 's execution cost $e_{1}$ (recall that pi-blocking is considered "bounded" only if it does not depend on job execution costs).

Priority boosting satisfies properties (i) and (iii) at the expense of (ii), whereas priority inheritance satisfies properties (ii) and (iii) at the expense of (i). For our purpose, however, we require a progress mechanism that satisfies both properties (i) and (ii), which according to Theorem 1 is only possible at the expense of (iii). As it turns out, such a mechanism is easily obtained by generalizing the idea of priority inheritance to "cluster inheritance," that is, inheritance should not only pertain to scheduling priorities, but also to where a job is eligible to execute, a concept that we refer to as "migratory priority inheritance" [14].

Def. 3. Let $W_{i}$ denote the set of jobs waiting for a job $J_{i}$ to release a resource. Under migratory priority inheritance, whenever $J_{i}$ is not scheduled (but ready) and there exists a job $J_{x} \in W_{i} \cup\left\{J_{i}\right\}$ such that $J_{x}$ is eligible to be scheduled in its assigned cluster (i.e., whenever $J_{i}$ is preempted and there are fewer than $c$ ready higher-effective-priority jobs in $J_{x}$ 's cluster), $J_{i}$ migrates to $J_{x}$ 's cluster (if necessary) and assumes $J_{x}$ 's priority. After releasing all locks, $J_{i}$ migrates back to its assigned cluster (if necessary).

Paraphrased, the idea is to move preempted, lock-holding jobs among clusters such that they are always local to a waiting job that would be scheduled if it were not suspended (if any). Migratory priority inheritance may be triggered whenever a lock-holding job $J_{i}$ is preempted, when a new waiter is added to $W_{i}$, or when one of the jobs in $W_{i}$ becomes one of the $c$ highest-effective-priority jobs in its cluster (due to the completion or suspension of a higher-priority job). If there are multiple candidates in $W_{i} \cup\left\{J_{i}\right\}$, then $J_{x}$ may be chosen arbitrarily among the candidates (e.g., cache locality may be taken into consideration).

Migratory priority inheritance is a straightforward generalization of classic priority inheritance [34]; it can also be understood as a greatly simplified, reduced-to-the-core ${ }^{4}$ version of Faggioli et al. multiprocessor bandwidth inheritance [24]. Notably, a progress mechanism akin to migratory priority inheritance appeared as early as 2001 in TU Dresden's Fiasco microkernel under the name "local helping" [26] (a more detailed discussion of these techniques can be found in [14]). While migratory priority inheritance has received surprisingly little attention in the real-time literature so far, it is ideal for our purposes as ready jobs are never pi-blocked since lock holders remain fully preemptive.

Lemma 1. In a mutual-exclusion semaphore protocol based on migratory priority inheritance, if a job $J_{i}$ is ready at time $t$, then it does not incur s-oblivious pi-blocking at time $t$.

Proof. Follows from the fact that priorities are not "duplicated" in a mutual-exclusion protocol. For each job that inherits a priority higher than $J_{i}$ 's base priority in cluster $C\left(T_{i}\right)$, there exists at least one suspended, pending job local

\footnotetext{
${ }^{4}$ The MBWI [24] also uses (non-optimal) FIFO queueing and allows for budget overruns, which is orthogonal to the idea of migrating lock holders.
} 
to $C\left(T_{i}\right)$ with base priority higher than $J_{i}$ 's base priority. If $J_{i}$ is ready but not scheduled at time $t$, then there exist $c$ jobs with higher effective priorities, which, due to the one-to-one inheritance relationship (and because priorities are inherited only locally after migrating to the blocked job's cluster), implies the existence of $c$ pending higher-base-priority jobs local to $C\left(T_{i}\right)$, which precludes s-oblivious pi-blocking.

However, migratory priority inheritance by itself is not sufficient to guarantee independence preservation. For example, if combined with the P-OMLP's contention token abstraction, requests of otherwise independent jobs could still conflict (i.e., contention tokens create artificial dependencies). Rather, a new locking protocol designed specifically to take advantage of migratory priority inheritance is required.

\subsection{Queue Structure for Optimal Waiting Times}

Asymptotic optimality under s-oblivious analysis requires $\max _{\tau} b_{i}=O(m)$. Clearly, this cannot be achieved using only FIFO queues, which would yield $\Omega(n)$ pi-blocking, nor can it be achieved using only priority queues, which are susceptible to starvation and can be shown to yield $\Omega(m \cdot n)$ pi-blocking [13]. However, a hybrid FIFO/priority queue design can be used to obtain $O(\mathrm{~m})$ pi-blocking bounds under global scheduling [12]. While such a hybrid queue cannot be directly applied to clustered scheduling (this would require comparing priorities across cluster boundaries, which, analytically, is meaningless), it can be used within each cluster. Further, while it is not possible to directly apply the P-OMLP's per-processor contention token design, it is beneficial to reuse the underlying idea of multiple "stages" of contention; that is, contention should be resolved first on an intra-cluster basis (where priorities can be safely compared) and only thereafter on an inter-cluster basis. Based on these considerations, the $O(m)$ independence-preserving locking protocol (OMIP) is defined as follows.

Structure. Each shared resource $\ell_{q}$ is protected by a global FIFO queue $\mathrm{GQ}_{q}$ of maximum length $\frac{m}{c}$. The job at the head of $\mathrm{GQ}_{q}$ holds $\ell_{q}$. Access to $\mathrm{GQ}_{q}$ is resolved on a per-cluster and per-resource basis: in each cluster $C_{k}$, there exist another two queues for each $\ell_{q}$, a bounded-length FIFO queue $\mathrm{FQ}_{q, k}$ of maximum length $c$ that feeds into $\mathrm{GQ}_{q}$, and a priority queue $\mathrm{PQ}_{q, k}$ that feeds into $\mathrm{FQ}_{q, k}$.

Rules. Requests for each $\ell_{q}$ are satisfied as follows. Let $J_{i}$ denote a job of a task assigned to cluster $C_{k}$. Conceptually, $J_{i}$ first enters its local $\mathrm{PQ}_{q, k}$ and then advances through the queues until it becomes the head of $\mathrm{GQ}_{q}$.

R1 When $J_{i}$ issues a request for $\ell_{q}$ and $\mathrm{FQ}_{q, k}$ is empty, then $J_{i}$ is enqueued in both $\mathrm{GQ}_{q}$ and $\mathrm{FQ}_{q, k}$. Otherwise, if there are fewer than $c$ jobs queued in $\mathrm{FQ}_{q, k}$, then $J_{i}$ is enqueued only in $\mathrm{FQ}_{q, k}$. Finally, if there are already $c$ jobs queued in $\mathrm{FQ}_{q, k}$, then $J_{i}$ is enqueued in $\mathrm{PQ}_{q, k}$.

$\mathbf{R 2} J_{i}$ 's request for $\ell_{q}$ is satisfied when it becomes the head of $\mathrm{GQ}_{q} . J_{i}$ is suspended while it waits (if necessary).

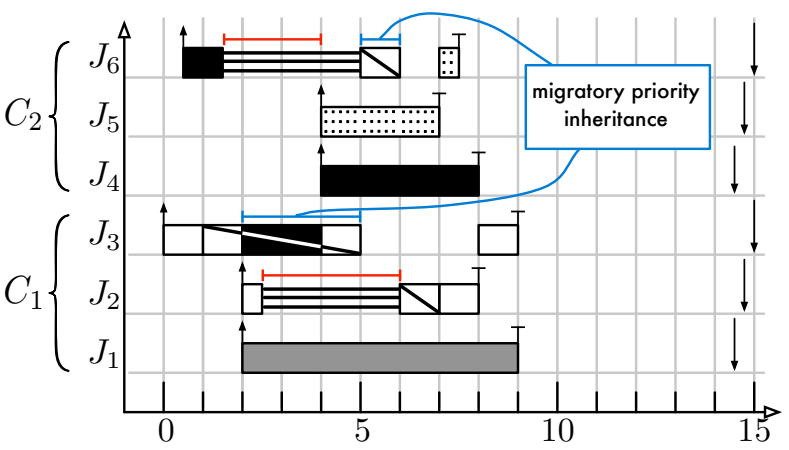

Figure 2: C-EDF schedule of six jobs in two two-processor clusters sharing one resource $\ell_{1}$ under the OMIP. Changes in shade indicate migrations; jobs $J_{3}$ and $J_{6}$ migrate across cluster boundaries due to migratory priority inheritance. See Fig. 1 for a legend.

R3 While $J_{i}$ holds $\ell_{q}$, it benefits from migratory priority inheritance (w.r.t. any job waiting to acquire $\ell_{q}$ ).

R4 When $J_{i}$ releases $\ell_{q}$, it is dequeued from both $\mathrm{GQ}_{q}$ and $\mathrm{FQ}_{q, k}$. If $\mathrm{PQ}_{q, k}$ is non-empty, then the head of $\mathrm{PQ}_{q, k}$ is transferred to $\mathrm{FQ}_{q, k}$. Further, if $\mathrm{FQ}_{q, k}$ is non-empty, then the new head of $\mathrm{FQ}_{q, k}$ is enqueued in $\mathrm{GQ}_{q}$. The new head of $\mathrm{GQ}_{q}$, if any, is resumed.

Rules R1-R4 together ensure $O(m)$ s-oblivious pi-blocking, which we show in Sec. 4.3 below after a brief example.

Example. Fig. 2 shows an example OMIP schedule under C-EDF scheduling with $m=4$ and $c=2$. Job $J_{3}$ enters both $\mathrm{GQ}_{1}$ and $\mathrm{FQ}_{1,1}$ and acquires $\ell_{1}$ at time 1 since $\ell_{1}$ is uncontested (Rule R1). Job $J_{6}$ requests $\ell_{1}$ shortly thereafter and enqueues in $\mathrm{GQ}_{1}$ and $\mathrm{FQ}_{1,2}$ (Rule R1), but must suspend since $J_{3}$ holds $\ell_{1}$ (Rule R2). At time $2, J_{3}$ is preempted due to the release of $J_{1}$ and $J_{2}$. As $J_{3}$ holds $\ell_{1}$, it is subject to migratory priority inheritance and migrates to $J_{6}$ 's cluster, where it is scheduled on processor 3 (Rule R3). $J_{2}$ proceeds to request $\ell_{1}$ as well and enqueues in $\mathrm{FQ}_{1,1}$, but suspends without enqueuing in $\mathrm{GQ}_{1}$ because $\mathrm{FQ}_{1,1}$ already contained $J_{3}$ (Rule R1). $J_{3}$ is now eligible to execute in $C_{1}$ again, but remains scheduled in $C_{2}$ since Def. 3 only takes effect when $J_{3}$ is preempted. At time 4 , jobs $J_{4}$ and $J_{5}$ are released in $C_{2}$, which implies that $J_{6}$ is no longer among the $c=2$ highest-priority pending jobs. $J_{3}$ is thus preempted and migrates back to $C_{1}$ (Def. 3), where it inherits $J_{2}$ 's priority (Rule R3). When $J_{3}$ releases $\ell_{1}$ at time 5 , it is removed from both $\mathrm{GQ}_{1}$ and $\mathrm{FQ}_{1,1}$, and the new head of $\mathrm{FQ}_{1,1}$, which is $J_{2}$, is enqueued in $\mathrm{GQ}_{1}$ (Rule R4). $J_{2}$ does not immediately acquire $\ell_{1}$ because it is preceded by $J_{6}$ (Rule 2 ). However, due to the presence of $J_{4}$ and $J_{5}, J_{6}$ is no longer of sufficient priority to be scheduled in $C_{2}$. Hence, migratory priority inheritance takes effect (Rule 3 ) and $J_{6}$ migrates to $C_{1}$ to execute its critical section. At time $6, J_{6}$ releases $\ell_{1}$ and ceases to benefit from migratory priority inheritance; it is thus preempted and not scheduled until time 7 when $J_{5}$ completes. $J_{2}$ finally acquires $\ell_{1}$ as it becomes the head of 
$\mathrm{GQ}_{1}$. Importantly, the example shows that jobs $J_{1}, J_{4}$, and $J_{5}$, which do not require $\ell_{1}$, do not incur any pi-blocking.

\subsection{PI-Blocking Optimality}

We bound the maximum s-oblivious pi-blocking incurred by a given task $T_{i}$ under the OMIP. In the following, let $J_{i}$ denote a job in cluster $C_{k}$ that has requested resource $\ell_{q}$. We begin with the observation that migratory priority inheritance ensures lock-holder progress.

Lemma 2. Let $J_{h}$ denote the job at the head of $G Q_{q}$ (i.e., the job that holds $\ell_{q}$ ). If $J_{i}$ incurs s-oblivious pi-blocking at time $t$, and if $J_{h}$ requires processor service at time $t$ (i.e., if $J_{h}$ is ready), then $J_{h}$ is scheduled.

Proof. Since $J_{i}$ incurs s-oblivious pi-blocking, it is among the $c$ highest-priority jobs in $C_{k}$. Therefore, $J_{i}$ would be eligible to execute if it were not suspended. By Rule R3, $J_{h}$ is subject to migratory priority inheritance. Thus, by Def. 3, if $J_{h}$ requires processor service to proceed (i.e., if $J_{h}$ is ready), then $J_{h}$ migrates to $C\left(T_{i}\right)$ (if required), inherits $J_{i}$ 's priority, and is thus scheduled by a processor in $C\left(T_{i}\right)$.

Lemma 2 implies that transitive pi-blocking due to processor unavailability is impossible, which allows us to bound maximum pi-blocking in terms of the number of critical sections that must complete before $J_{i}$ acquires $\ell_{q}$. Due to the multi-stage structure of the OMIP, we proceed in reverse order, starting with jobs already enqueued in $\mathrm{GQ}_{q}$.

Lemma 3. Once $J_{i}$ is enqueued in $G Q_{q}$, at most $\frac{m}{c}-1$ requests for $\ell_{q}$ must complete before $J_{i}$ holds $\ell_{q}$.

Proof. Due to the interplay of Rules R1 and R4, at most one job per cluster is enqueued in $\mathrm{GQ}_{q}$ at any time. Thus, as there are $\frac{m}{c}$ clusters and since $\mathrm{GQ}_{q}$ is FIFO-ordered, at most $\frac{m}{c}-1$ critical sections must complete before $J_{i}$ becomes the head of the queue and its request is satisfied (Rule 2).

This also bounds how long $J_{i}$ remains the head of $\mathrm{FQ}_{q, k}$.

Lemma 4. Once $J_{i}$ becomes the head of $\mathrm{FQ}_{q, k}$, at most $\frac{m}{c}$ requests for $\ell_{q}$ must complete before $J_{i}$ is dequeued.

Proof. Follows from Lemma 3 and the fact that $J_{i}$ must complete its own request before being dequeued.

This yields a bound on how long it takes to traverse $\mathrm{FQ}_{q, k}$.

Lemma 5. Once $J_{i}$ is enqueued in $F Q_{q, k}$, at most $\frac{m}{c} \cdot(c-1)$ requests for $\ell_{q}$ must complete before $J_{i}$ enters $G Q_{q}$.

Proof. $J_{i}$ enters $\mathrm{GQ}_{q}$ when it becomes the head of $\mathrm{FQ}_{q, k}$ (Rules R1 and R4). Due to the bounded length of $\mathrm{FQ}_{q, k}$, at most $c-1$ jobs precede $J_{i}$ in $\mathrm{FQ}_{q, k}$. By Lemma 4, each such job, once it has become the head of $\mathrm{FQ}_{q, k}$ is dequeued after at most $\frac{m}{c}$ requests complete. Thus there are no jobs remaining in front of $J_{i}$ after $\frac{m}{c} \cdot(c-1)$ requests complete.

The final step is to bound the pi-blocking that $J_{i}$ incurs while waiting in $\mathrm{PQ}_{q, k}$. Since priority queues do not offer strong progress guarantees, simply counting preceding jobs is insufficient in this case and an indirect proof is required.
Lemma 6. While $J_{i}$ waits in $P Q_{q, k}$, at most $m$ requests for $\ell_{q}$ must complete until either $J_{i}$ enters $F Q_{q, k}$ or $J_{i}$ stops to incur s-oblivious pi-blocking.

Proof. Suppose not. Then there exists a point in time $t$ at which more than $m$ requests have completed since $J_{i}$ entered $\mathrm{PQ}_{q, k}$ and $J_{i}$ still waits in $\mathrm{PQ}_{q, k}$ and incurs pi-blocking. By Lemma 4, a job at the head of $\mathrm{FQ}_{q, k}$ is dequeued after at most $\frac{m}{c}$ requests complete. Thus, at time $t$, at least $m / \frac{m}{c}=c$ jobs have been dequeued from $\mathrm{FQ}_{q, k}$ since $J_{i}$ entered $\mathrm{PQ}_{q, k}$. By Rule R4, the highest-priority job in $\mathrm{PQ}_{q, k}$ is transferred to $\mathrm{FQ}_{q, k}$ when the head of $\mathrm{FQ}_{q, k}$ is dequeued. Since $J_{i}$ is still in $\mathrm{PQ}_{q, k}$ at time $t$, a job with higher priority has been moved to $\mathrm{FQ}_{q, k}$ at least $c$ times. Therefore, each job in $\mathrm{FQ}_{q, k}$ has a higher priority than $J_{i}$, that is, there exist $c$ higherpriority pending jobs in cluster $C_{k}$ at time $t$, which precludes s-oblivious pi-blocking (recall Def. 1). Contradiction.

Having bounded maximum pi-blocking during each of the OMIP's "stages" that a job traverses, we obtain the following overall bound on per-resource s-oblivious pi-blocking.

Lemma 7. $J_{i}$ incurs at most $b_{i, q}=N_{i, q} \cdot(2 m-1) \cdot L_{q}^{\max }$ s-oblivious pi-blocking due to requests by any task for $\ell_{q}$.

Proof. By Lemma $1, J_{i}$ incurs pi-blocking only while suspended. Consider a single request for $\ell_{q}$. While $J_{i}$ is suspended waiting to acquire $\ell_{q}$, the amount of pi-blocking incurred is determined by the number of conflicting requests that precede $J_{i}$ 's request. By Lemma 6 , at most $m$ requests must complete before $J_{i}$ enters $\mathrm{FQ}_{q, k}$ or ceases to incur pi-blocking in $\mathrm{PQ}_{q, k}$. By Lemmas 3 and 5, at most $\frac{m}{c}-1+\frac{m}{c} \cdot(c-1)=m-1$ requests must complete from the time that $J_{i}$ enters $\mathrm{FQ}_{q, k}$ until it holds $\ell_{q}$. Lemma 2 implies that the job holding $\ell_{q}$ progresses towards completion of its request whenever $J_{i}$ incurs s-oblivious pi-blocking. Thus, after $J_{i}$ has incurred $(m+m-1) \cdot L_{q}^{\max }$ s-oblivious piblocking, at least $2 m-1$ requests must have completed and $J_{i}$ holds $\ell_{q}$. The stated bound follows from considering the maximum number of requests issued by any $J_{i}$ for $\ell_{q}$.

Note that Lemma 7 implies that the OMIP is indeed independence-preserving (recall Def. 2). Finally, we obtain the following bound on total s-oblivious pi-blocking.

Theorem 2. Under clustered JLFP scheduling with the OMIP, a job $J_{i}$ incurs in total at most $b_{i}=\sum_{q} b_{i, q}=$ $\sum_{q} N_{i, q} \cdot(2 m-1) \cdot L_{q}^{\max }=O(m)$ s-oblivious pi-blocking. Proof. Follows from Lemma 7 since $b_{i}=\sum_{q} b_{i, q}$.

Prior work has established $m-1$ as a simple per-request lower bound under s-oblivious analysis [12]; the OMIP is thus optimal within a factor of $\frac{2 m-1}{m-1}=2+\frac{1}{m-1} \approx 2$.

\section{Implementation and Evaluation}

We modified the existing C-EDF plugin in LITMUS ${ }^{\mathrm{RT}}$ [1], a real-time extension of the Linux kernel, to incorporate migratory priority inheritance, the OMIP, and the OMLP. The OMIP's multi-level queue structure is easily realized by 
combining multiple wait queues, which are readily available in Linux. Most complexity stems from the need to coordinate scheduling decisions across cluster boundaries when a job is eligible to be scheduled in multiple clusters due to migratory priority inheritance. In particular, if a processor decides to not schedule a lock-holding job $J_{l}$ because it observes that $J_{l}$ is already scheduled in another cluster, the processor must be notified if $J_{l}$ is subsequently preempted. In our prototype, this is realized with a per-semaphore bitmask that tracks on which processors a job holding the semaphore may currently be scheduled, and by sending an inter-processor interrupt (IPI) to available processors when a lock-holder preemption occurs. A migration, if possible, is then carried out by the first available processor to reschedule in response to the IPI. A detailed discussion of the implementation is omitted here due to space constraints; however, our changes are publicly available as a patch on the LITMUS ${ }^{\mathrm{RT}}$ homepage [1].

\subsection{Response-Time Measurements}

Based on LITMUS ${ }^{\mathrm{RT}}$, we conducted an experiment to empirically demonstrate that the OMIP is effective at protecting latency-sensitive tasks, that is, to rule out the possibility that independence-preservation is merely an "analytical trick" without practical impact. To this end, we configured a simple synthetic task set on a $2.0 \mathrm{GHz}$ Intel Xeon X7550 system with $m=8$ cores using the modified LITMUS ${ }^{\text {RT }}$ C-EDF plugin with clusters defined by the L1 cache topology, which results in P-EDF scheduling $(c=1)$ on the test platform.

On each core, we launched four tasks with periods $1 \mathrm{~ms}$, $25 \mathrm{~ms}, 100 \mathrm{~ms}$, and $1000 \mathrm{~ms}$ and execution costs of roughly $0.1 \mathrm{~ms}, 2 \mathrm{~ms}, 15 \mathrm{~ms}$, and $600 \mathrm{~ms}$, resp. The latency-sensitive, one-millisecond tasks did not access any shared resources. All other tasks shared a single lock with an associated maximum critical section length of approximately $L^{\max }=1 \mathrm{~ms}$, and each of their jobs acquired the lock once. While the task set is synthetic, the chosen parameters are inspired by the range of periods found in automotive systems [16, 37] and we believe them to be a reasonable approximation of heterogenous timing constraints as they arise in practice.

We ran the task set once using the C-OMLP, once using the OMIP, and once using no locks at all (as a baseline assuming that all tasks are independent) for 30 minutes each. We traced the resulting schedules using LITMUS $^{\mathrm{RT}}$ 's sched_trace facility and recorded the response times of more than 45, 000, 000 individual jobs. Fig. 3 shows two histograms of recorded response times under each configuration. Fig. 3(a) depicts response times of the one-millisecond tasks. Due to the short period (and consistent deadline tie-breaking), their jobs always have the highest priority and thus incur no delays at all in the absence of locks (all response times are in the first bin). In contrast, under the C-OMLP, jobs are not protected from pi-blocking due to unrelated critical sections and response times exceeding $8 \mathrm{~ms}$ were observed-priority boosting causes deadline misses in latency-sensitive tasks

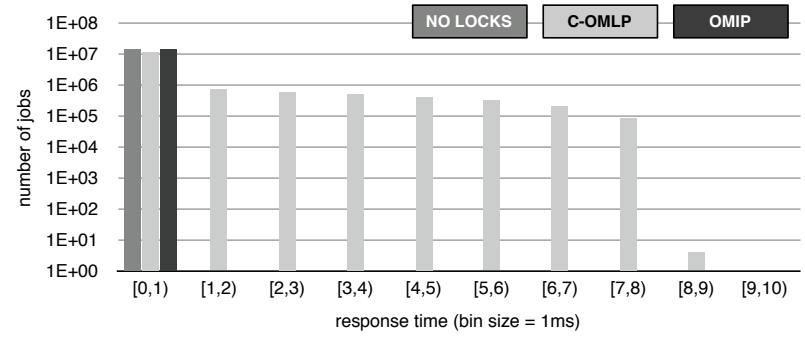

(a) Response times of latency-sensitive tasks with period $p_{i}=1 \mathrm{~ms}$.

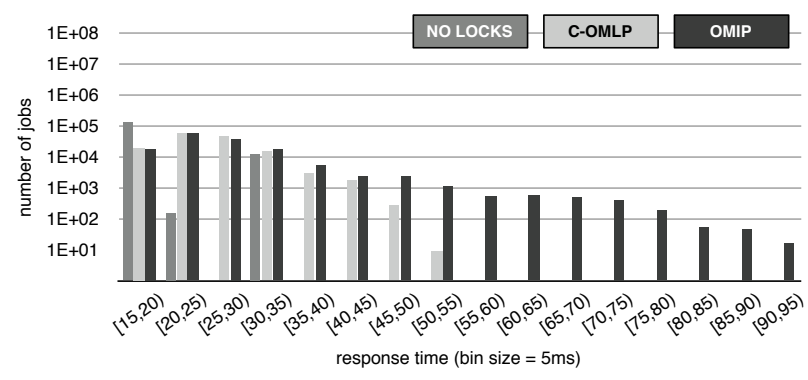

(b) Response times of regular tasks with period $p_{i}=100 \mathrm{~ms}$.

Figure 3: Response times measured in LITMUS ${ }^{\mathrm{RT}}$ under C-EDF scheduling with the OMIP, the C-OMLP, and without locks.

(not coincidentally, $8 m s \approx m \cdot L^{\max }$ ). Not so under the OMIP, where the response-times are identical to the case without locks - task independence was indeed preserved.

However, mutual exclusion invariably causes some delays, and if such delays do not manifest in the highest-priority jobs, then they will be necessarily observable elsewhere. This is apparent in Fig. 3(b), which depicts the responsetime distribution of the $100 \mathrm{~ms}$-tasks. For these tasks with considerable slack, worst-case response-times are noticeably shorter under the C-OMLP than under the OMIP. This emphasizes that there is an obvious tradeoff between not penalizing higher-priority jobs and rapidly completing critical sections. To explore this tradeoff, we conducted schedulability experiments, which allow analytical differences w.r.t. response-time guarantees to be quantified (as opposed to the differences in observed response times discussed so far).

\subsection{Schedulability Experiments}

In preparation, we derived and implemented fine-grained (i.e., non-asymptotic) pi-blocking analysis of the OMIP suitable for schedulability analysis based on a recently developed analysis technique using linear programming [11]; details can be found online [10]. To quantify each protocol's impact on schedulability, we generated task sets using Emberson et al.'s method [22] consisting of $n \in\{20,30,40\}$ tasks with total utilization $U \in\{0.4 m, 0.5 m, 0.7 m\}$. Of the $n$ tasks, $n^{\text {lat }} \in\{0,1, \ldots, 8\}$ were chosen to be latency-sensitive. Latency-sensitive tasks were assigned a period randomly chosen from $[0.5 \mathrm{~ms}, 2.5 \mathrm{~ms}]$, and regular tasks were assigned 


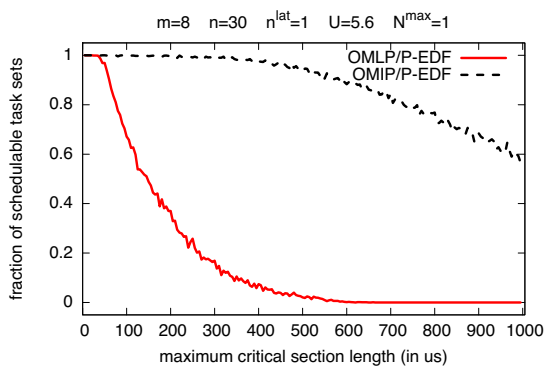

(a) A single latency-sensitive task dominates.

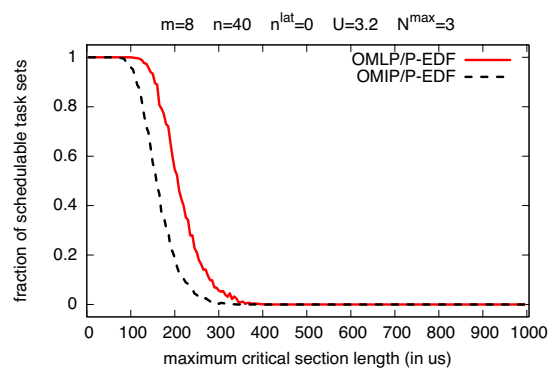

(b) Overall pi-blocking dominates.

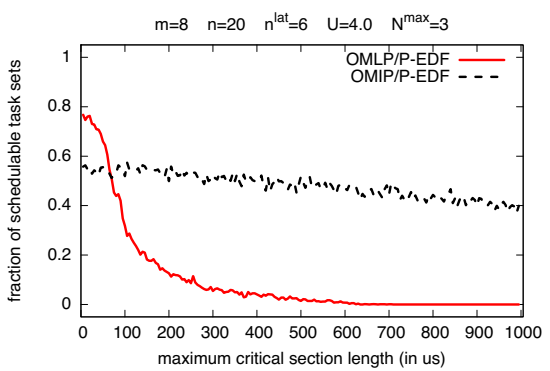

(c) Combination of effects.

Figure 4: The effect of increasing maximum critical section length under P-EDF scheduling with the C-OMLP and the OMIP.

a period randomly chosen from $[10 \mathrm{~ms}, 1000 \mathrm{~ms}]$ (in both cases with a granularity of $500 \mu \mathrm{s}$ ).

The $n^{\text {lat }}$ latency-sensitive tasks shared three resources, which each job accessed once each with maximum critical section lengths chosen uniformly from $[1 \mu s, 15 \mu s]$. The remaining $n-n^{\text {lat }}$ regular tasks shared 12 different resources, of which each task accessed $N^{\max } \in\{1,2,3\}$, with a maximum critical section length randomly chosen from $[1, m c s l]$, where $m c s l$ is a variable parameter denoting the maximum critical section length. We varied $m c s l \in[5 \mu s, 1000 \mu s]$ in steps of $5 \mu \mathrm{s}$ on processor platforms with $m \in\{2,4,8,16\}$ processors and generated for each combination of $n, n^{\text {lat }}$, $U, N^{\max }$, and mcsl at least 1000 task sets (more than $150,000,000$ in total). Each generated task set was schedulability tested under both the C-OMLP and the OMIP assuming P-EDF scheduling (i.e., C-EDF with $c=1$ ). The full set of results, 678 schedulability plots when visualized as a function of $m c s l$, is available online [10]; three illustrative examples highlighting two main "bottlenecks" that constrain schedulability are shown in Fig. 4.

Fig. 4(a) shows one of many cases where the OMIP is clearly preferable. In this scenario with one latency-sensitive task (i.e., $n^{\text {lat }}=1$, see figure for all parameters), schedulability under the C-OMLP decreases rapidly as critical section lengths start to exceed $\approx 50 \mu s$. The cause is that starting with $m c s l=50 \mu s$, latency-sensitive tasks with a period of $p_{i}=500 \mu \mathrm{s}$ are likely rendered unschedulable by delays due to unrelated critical sections in the range of $m \cdot L^{\max } \approx 50 \mu \mathrm{s}=400 \mu \mathrm{s}$, similar to the hand-crafted example discussed in Sec. 5.1. In contrast, the OMIP achieves high schedulability even in the presence of much longer critical sections. In this scenario, latency sensitivity is the bottleneck, and the OMIP is preferable for such workloads.

In contrast, Fig. 4(b) shows a scenario with the opposite outcome. In this case, no latency-sensitive tasks are included in the generated task sets $\left(n^{\text {lat }}=0\right)$ and there is a high degree of contention $\left(N^{\max }=3\right)$. As a result, independencepreservation is not essential and constant factors in the piblocking analysis dominate: while the C-OMLP and the OMIP are both $O(m)$ protocols, the C-OMLP has a perrequest bound of $(m-1) \cdot L^{\max }$ [13], whereas the OMIP has a per-request bound of $(2 m-1) \cdot L^{\max }$ (Lemma 7). The C-OMLP thus achieves somewhat higher schedulability in this case, though long critical sections are infeasible under either protocol due to the high level of contention.

Finally, Fig. 4(c) shows a scenario with $n^{\text {lat }}=6$ and $N^{\max }=3$ where both effects combine. Due to the high level of contention, schedulability under the OMIP is limited across the entire range of critical section lengths, whereas under the C-OMLP, schedulability for short critical sections is markedly higher than under the OMIP due to the C-OMLP's advantage in constant factors, but then decreases rapidly due to the lack of independence preservation under the C-OMLP.

In summary, our results show that independence preservation and the OMIP are clearly beneficial for latency-sensitive workloads. Further, the OMIP is competitive with and often performs similar to the C-OMLP even if independence preservation is not required, but may perform worse than the $\mathrm{C}$-OMLP in the presence of heavy contention.

\section{Conclusion}

This paper is the first to investigate the challenge of supporting latency-sensitive real-time applications under partitioned and clustered scheduling. We have proposed independence preservation to capture the essential requirement of such workloads. The primary contribution of this paper is the OMIP, the first independence-preserving real-time semaphore protocol for clustered JLFP scheduling. Interestingly, the OMIP employs a novel three-stage queue, which yields asymptotic optimality under s-oblivious analysis.

In summary, latency-sensitive real-time applications have long been well-supported on uniprocessors, but could not be hosted on partitioned multiprocessors with prior semaphore protocols. The OMIP is the first semaphore protocol to overcome this limitation, at the expense of job migrations.

Naturally, job migrations do cause additional overheads, and current industry practice thus regards them critically. However, if job migration is disallowed or fundamentally infeasible (e.g., if the processors in different clusters implement incompatible instruction sets), then Theorem 1 shows that there is little that can be done besides disallowing crosscluster resource sharing altogether. In contrast, in shared- 
memory systems in which global scheduling is technically possible, but a non-global scheduling approach is preferred for other reasons (e.g., to improve cache affinity or to reuse existing uniprocessor algorithms), the OMIP is a viable compromise: first, migratory priority inheritance is triggered only if the lock-holding job is preempted, which means that its cache contents are likely evicted anyway, and second, it is cheaper to migrate critical sections than it is to migrate jobs since the cache footprint of a critical section is typically much smaller than that of an entire job. Concerning the number of times that a critical section may be preempted, migratory priority inheritance is no worse in this regard than the widely deployed PIP. Nontheless, it will be interesting to study the effects of the OMIP on overheads in more detail.

In future algorithmic work, we seek to lift the assumption that jobs lock only one resource at a time. Ward and Anderson [38] recently showed how to support nested critical sections without loss of asymptotic optimality. However, under s-oblivious analysis, their approach permits at most $m$ concurrent (outermost) critical sections by means of a global token lock, which can serialize otherwise independent jobs. Ward and Anderson's technique [38] thus preserves asymptotic optimality when applied to the OMIP, but it is not independence-preserving. However, it may be possible to combine Ward and Anderson's approach with the FMLP's notion of resource groups [7] to obtain independence preservation and asymptotic optimality despite lock nesting.

Finally, we further seek to explore the design space of independence-preserving locking protocols for s-aware analysis, and are currently investigating synchronization mechanisms that provide "freedom from interference" (i.e., temporal isolation) among mutually untrusting tasks, despite resource sharing in the presence of budget overruns, for which the OMIP is an essential building block [9].

\section{References}

[1] The LITMUS ${ }^{R T}$ project. http: / / www. litmus-rt.org.

[2] J. Anderson, R. Jain, and K. Jeffay. Efficient object sharing in quantum-based real-time systems. In Proc. of the 19th Real-Time Systems Symposium, 1998.

[3] T. Baker. Stack-based scheduling for realtime processes. Real-Time Systems, 3(1):67-99, 1991.

[4] T. Baker and S. Baruah. Schedulability analysis of multiprocessor sporadic task systems. In Handbook of Real-Time and Embedded Systems. Chapman Hall/CRC, 2007.

[5] S. Baruah. Techniques for multiprocessor global schedulability analysis. In Proc. of the 28th Real-Time Systems Symposium, 2007.

[6] M. Bertogna and M. Cirinei. Response-time analysis for globally scheduled symmetric multiprocessor platforms. In Proc. of the $28 \mathrm{th}$ Real-Time Systems Symposium, 2007.

[7] A. Block, H. Leontyev, B. Brandenburg, and J. Anderson. A flexible real-time locking protocol for multiprocessors. In Proc. of the 13th Conference on Embedded and Real-Time Computing Systems and Applications, 2007.

[8] B. Brandenburg. Scheduling and Locking in Multiprocessor Real-Time Operating Systems. PhD thesis, The University of North Carolina at Chapel Hill, 2011.

[9] B. Brandenburg. Virtually exclusive resources. Technical Report MPI-SWS-2012-005, MPI-SWS, May 2012.

[10] B. Brandenburg. A fully preemptive multiprocessor semaphore protocol for latency-sensitive real-time applications (extended version). Technical Report MPI-SWS-2013-003, MPI-SWS, May 2013.

[11] B. Brandenburg. Improved analysis and evaluation of real-time semaphore protocols for P-FP scheduling. In Proc. of the 19th RealTime and Embedded Technology and Applications Symposium, 2013.

[12] B. Brandenburg and J. Anderson. Optimality results for multiprocessor real-time locking. In Proc. of the 31st Real-Time Systems Symposium, 2010 .

[13] B. Brandenburg and J. Anderson. The OMLP family of optimal multiprocessor real-time locking protocols. Design Automation for Embedded Systems, online first, July 2012.

[14] B. Brandenburg and A. Bastoni. The case for migratory priority inheritance in Linux: Bounded priority inversions on multiprocessors. In Proc. of the 14th Real-Time Linux Workshop, 2012.

[15] B. Brandenburg, J. Calandrino, A. Block, H. Leontyev, and J. Anderson. Synchronization on real-time multiprocessors: To block or not to block, to suspend or spin? In Proc. of the 14th Real-Time and Embedded Technology and Apps. Symposium, 2008.

[16] D. Buttle. Real-time in the prime-time. Keynote presentation at the 24th Euromicro Conference on Real-Time Systems, July 2012.

[17] J. Calandrino, J. Anderson, and D. Baumberger. A hybrid real-time scheduling approach for large-scale multicore platforms. In Proc. of the 19th Euromicro Conference on Real-Time Systems, 2007.

[18] Y. Chang, R. Davis, and A. Wellings. Reducing queue lock pessimism in multiprocessor schedulability analysis. In Proc. of the 18th Conference on Real-Time and Network Systems, 2010.

[19] A. Chou, J. Yang, B. Chelf, S. Hallem, and D. Engler. An empirical study of operating systems errors. In Proc. of the 18th Symposium on Operating Systems Principles, 2001.

[20] T. Craig. Queuing spin lock algorithms to support timing predictability. In Proc. of the 14th IEEE Real-Time Systems Symposium, 1993.

[21] A. Easwaran and B. Andersson. Resource sharing in global fixedpriority preemptive multiprocessor scheduling. In Proc. of the 30th Real-Time Systems Symposium, 2009.

[22] P. Emberson, R. Stafford, and R. Davis. Techniques for the synthesis of multiprocessor tasksets. In 1st Workshop on Analysis Tools and Methodologies for Embedded and Real-time Systems, 2010.

[23] Express Logic, Inc. ThreadX real-time operating system. http: //rtos. com/products/threadx/, accessed 1/26/2013.

[24] D. Faggioli, G. Lipari, and T. Cucinotta. The multiprocessor bandwidth inheritance protocol. In Proc. of the 22nd Euromicro Conference on Real-Time Systems, 2010.

[25] P. Gai, M. di Natale, G. Lipari, A. Ferrari, C. Gabellini, and P. Marceca. A comparison of MPCP and MSRP when sharing resources in the Janus multiple processor on a chip platform. In Proc. of the 9th Real-Time And Embedded Technology Application Symposium, 2003.

[26] M. Hohmuth and M. Peter. Helping in a multiprocessor environment. In Proceeding of the Second Workshop on Common Microkernel System Platforms, 2001.

[27] G. Macariu and V. Cretu. Limited blocking resource sharing for global multiprocessor scheduling. In Proc. of the 23 rd Euromicro Conference on Real-Time Systems, 2011

[28] F. Nemati, M. Behnam, and T. Nolte. Independently-developed realtime systems on multi-cores with shared resources. In Proc. of the 23rd Euromicro Conference on Real-Time Systems, 2011.

[29] Open Source Automation Development Lab eG. Continuous worst-case latency monitoring. https://WwW. osadl.org/Continuous-latency-monitoring. qa-farm-monitoring.0.html, accessed 1/26/2013.

[30] R. Rajkumar. Real-time synchronization protocols for shared memory multiprocessors. In Proc. of the 10th Conference on Dist Computing Systems, 1990

[31] R. Rajkumar. Synchronization In Real-Time Systems-A Priority Inheritance Approach. Kluwer Academic Publishers, 1991.

[32] R. Rajkumar, L. Sha, and J. Lehoczky. Real-time synchronization protocols for multiprocessors. In Proc. of the 9th Real-Time Systems Symposium, 1988.

[33] F. Ridouard, P. Richard, and F. Cottet. Negative results for scheduling independent hard real-time tasks with self-suspensions. In Proc. of 25th Real-Time Systems Symposium, 2004.

[34] L. Sha, R. Rajkumar, and J. Lehoczky. Priority inheritance protocols: an approach to real-time synchronization. IEEE Trans. Comput., 39(9):1175-1185, 1990.

[35] H. Takada and K. Sakamura. Predictable spin lock algorithms with preemption. In Proc. of the 11th Workshop on Real-Time Operating Systems and Software, 1994.

[36] H. Takada and K. Sakamura. A novel approach to multiprogrammed multiprocessor synchronization for real-time kernels. In Proc. of the 18th Real-Time Systems Symposium, 1997.

[37] K. Tindell and A. Burns. Guaranteeing message latencies on control area network (CAN). In Proc. of the 1st Intl. CAN Conference, 1994.

[38] B. Ward and J. Anderson. Supporting nested locking in multiprocessor real-time systems. In Proc. of the 24th Euromicro Conference on RealTime Systems, 2012. 